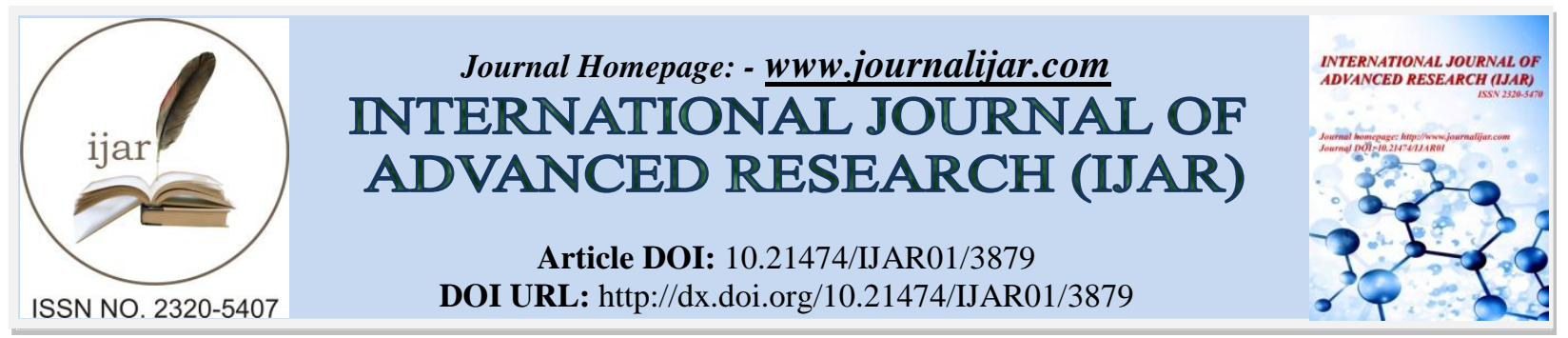

RESEARCH ARTICLE

\title{
ELECTROENCEPHALOGRAPHIC CHANGES IN TYPE 2 DIABETES MELLITUS DURING HYPERVENTILATION AND POST-HYPERVENTILATION
}

Raju Panta ${ }^{1}$, Bishnu Hari Paudel ${ }^{2}$, and Nirmala Limbu ${ }^{2}$

1. Department of Physiology, Trinity School of Medicine, St. Vincent and the Grenadines.

2. Department of Basic \& Clinical Physiology, B.P. Koirala Institute of Health Sciences, Nepal.

\section{Manuscript Info}

Manuscript History

Received: 06 February 2017

Final Accepted: 05 March 2017

Published: April 2017

Key words:-

EEG, Diabetes mellitus, Neuropathy,

Hyperventilation, CNS abnormalities

\section{Abstract}

Diabetes mellitus is the most common metabolic disorder that results in various complications including the brain abnormalities. Hyperventilation (HV) is a classic activation procedure during routine electroencephalography (EEG) recording that increases the yield of electrical activity generated in the cerebral cortex.

Objectives: To investigate the electroencephalographic (EEG) changes in type 2 diabetes mellitus (T2DM) patients during hyperventilation (HV) and post-HV.

Methods: The 16-channel EEG recording of 35 male patients with T2DM on oral hypoglycemic medication and 35 age- and sex-matched healthy subjects (controls) were done during and after hyperventilation for 3 minutes each. The quantitative EEG analysis was done using Fast Fourier Transformation (FFT). The EEG power spectra were subjected to log-transformation and compared using student's t-test.

Results: The T2DM patients had diffuse increase in low-frequency slow waves at delta range $(\mathrm{p}<0.05)$ and high-frequency fast waves at beta range $(\mathrm{p}<0.05)$ as compared to the controls in EEG during HV. There was a prolonged existence of delta activity at few brain regions $(\mathrm{p}<0.05)$ and beta activity in some brain regions $(\mathrm{p}<0.05)$ in the EEG after cessation of HV during post-HV.

Conclusion: The increased yield of EEG activity at delta and beta ranges in T2DM patients during $\mathrm{HV}$ and the prolonged existence of these responses after cessation of $\mathrm{HV}$ are suggestive of central nervous system (CNS) abnormalities in T2DM patients.

Copy Right, IJAR, 2017. All rights reserved.

\section{Introduction:-}

Diabetes mellitus (DM) remains most common metabolic disorder, with an estimated 415 million adults affected by diabetes worldwide (1). A complex interaction of direct and indirect metabolic consequences of insulin deficiency and additional genetic and environmental factors result in chronic complications affecting nephrons, blood vessels, retina, and neurons (2).

Several studies have reported the manifestations of cerebral disorders in type 2 diabetes mellitus (T2DM) at neurochemical, electrophysiological, structural, and neurobehavioral levels $(3,8,9)$. The alterations in cerebral 
blood supply, metabolic derangements, recurrent episodes of hypoglycemia, and poor metabolic control also affect the brain (3). A bidirectional relationship exists between diabetes mellitus (DM) and major depressive disorder (MDD) (4). Delirium, substance use disorders, depression, anxiety, and psychotic illnesses like schizophrenia, and eating disorders are some of the psychiatric disorders particularly relevant with regard to DM (5).

Electroencephalography (EEG) is a tool for recording spontaneous electrical activity generated in the cerebral cortex using multiple electrodes placed on the scalp. EEG signal is a reflection of electrical currents flowing in the extracellular space generated by the algebraic summation of excitatory and inhibitory postsynaptic potentials occurring on many cortical neurons. The scalp electrodes pick up the summated excitatory postsynaptic potential (EPSP) and inhibitory postsynaptic potential (IPSP) conducted through the skull. One estimate suggests that 6 square centimeters $\left(\mathrm{cm}^{2}\right)$ of cortical surface area must be synchronously activated for a potential to be recorded at the scalp $(6,7)$. Quantitative EEG (QEEG) is a more advanced form of EEG, which converts electrical signals from the brain to digital form. This reveals the EEG patterns undetectable by the naked eye (7).

Hyperventilation (HV) is a classic activation procedure during routine EEG recording that increases the yield of EEG. The subjects are usually asked to hyperventilate for 3 minutes, at the rate of 20 breaths per minute. It usually activates the three-per-second spike and wave discharge of primary generalized epilepsy $(6,7)$.

Patients with T2DM have increased EEG activity in low-frequency slow waves and high-frequency fast waves indicative of diffuse central neuropathy (8). Baskaran et al reported similar neurophysiological abnormalities in both DM and MDD, including elevated EEG activity in low-frequency slow waves (9). The recurrent episodes of hypoglycemia enhance the varying brain electrical responses to HV in different subjects. A buildup of diffuse slow activity occurs 30 to 60 seconds after the conclusion of HV. These changes occur due to a fall in partial pressure of carbon dioxide $\left(\mathrm{PCO}_{2}\right)$ in arterial blood, which leads to cerebral vasoconstriction and thus to reduced cerebral blood flow. The prolonged existence of any response in EEG after cessation of HV is abnormal (10). Some studies have reported central neuropathy in DM as evidenced by EEG changes $(3,8)$. To the best of our knowledge, very few studies have recorded EEG changes in DM during HV. In our study, we provoked the brain activity in T2DM patients by voluntary $\mathrm{HV}$ to reveal the effects of $\mathrm{HV}$ on brain activities of T2DM patients during EEG recording.

\section{Material and Methods:-}

This was a comparative cross-sectional study conducted on 35 male patients with T2DM and 35 age- and sexmatched controls. The subjects were selected using a convenience sampling technique based on the inclusion and exclusion criteria. The T2DM patients were newly diagnosed or follow-up cases on oral hypoglycemic medication including sulphonylureas and metformin. The controls had no symptoms of any diseases, normal on clinical examination, normal fasting blood sugar (FBS), age- and sex-matched, were not on any medication, and without any clinical evidence of infectious, systemic, metabolic, and neuropsychiatric illnesses.

The participants were familiarized with the laboratory setting and the study design. All the participants were informed of the potential risks and recording procedures, and informed written consent was obtained. They were interviewed using standard questionnaires for their medical history. Their detailed medical history, clinical examinations, and health status were documented using standard case history sheets. The recent reports on FBS, post-prandial blood sugar (PPBS), and glycated hemoglobin $\left(\mathrm{HbA}_{1} \mathrm{C}\right)$ of the T2DM patients were noted. The FBS of controls was assessed and documented. The weight, height, systolic blood pressure (SBP), diastolic blood pressure (DBP), respiratory rate (RR), and heart rate (HR) of both the T2DM patients and controls were measured using standard tools and methods. Their body mass index (BMI) was calculated using the standard formula, i.e., BMI = weight in kilogram $(\mathrm{kg}) /$ height in square meter $\left(\mathrm{m}^{2}\right)$. The digital EEG machine (Nihon Kohden- Neurofax: optiplex GXMT5120) was used for 16-channel EEG recording.

\section{EEG Recording:-}

Each subject was advised to come with clean scalp and hair, with proper last night's sleep (minimum 6 hours) and after 2 hours of light breakfast. In addition, the subjects were suggested not to drink tea/coffee 24 hours before the test. The temperature of the lab was maintained at $26 \pm 2^{\circ} \mathrm{C}$. All the equipment was checked prior to the recording procedure. They were advised to be relaxed and their comfort was maintained before and during the recording procedures. All the recordings were done between 10-11:30 a.m. to minimize the variation due to time.

Each subject is allowed to take rest for 10 minutes in the supine position on the dental chair after adjusting the head and back of the chair according to subject's comfort. The International 10-20 common average reference system was used for scalp electrode placement. The EEG recording was undertaken in a quiet, relaxed environment, with the 
subject lying comfortably in supine position. The 16-channel EEG was recorded first for 6 minutes at rest during eyes-open and eyes-close conditions for 3 minutes each. Then the subject was asked to hyperventilate for 3 minutes, at the rate of 20 breaths per minute. The EEG recorded during HV and post-HV for 3 minutes each was considered for QEEG analysis.

\section{EEG Data Acquisition and Analysis:-}

EEG waveforms were reduced and analyzed by using Focus software version (1.1). Initially, the records were visually inspected on the computer screen to check for artifacts due to eye blinks, and detectable eye and body movements. After visual inspection, three artifact-free-5-second epochs were selected from just before the end of $1^{\text {st }}$, $2^{\text {nd }}$ and $3^{\text {rd }}$ minutes of EEG recording during HV, and post-HV. Thereafter, Fast Fourier Transformation (FFT) method was applied to these data for decomposition of EEG waveforms into sine wave components in terms of respective frequencies.

These components were used to estimate the spectral power in square microvolt $\left(\mu \mathrm{V}^{2}\right)$ for frequency in the ranges of delta $(0.5-4.0 \mathrm{~Hz})$, theta $(4.0-7.0 \mathrm{~Hz})$, alpha1 (7.0-10.0 Hz), alpha2 (10.0-13.0 Hz), and beta (13.0-32.0 Hz) bands. The spectral power for each band, thus obtained from different regions of the brain was exported to Microsoft Excel worksheet for further analysis. The powers from three epochs were averaged for each subject. The data obtained from the T2DM patients and controls were compared.

\section{Statistical Analysis:-}

The data obtained were exported to Statistical Package for the Social Sciences (SPSS version 18) and were tested for normal distribution. The data of age, weight, height, BMI, SBP, DBP, HR, RR, and FBS were normally distributed and expressed in terms of mean \pm standard deviation (SD). The data of EEG power spectra were subjected to statistical analysis after log transformation as they were non-normally distributed. The EEG power spectra were expressed as mean \pm standard deviation. The Unpaired t-test was applied to compare the variables between the T2DM patients and controls. A $p$ value of $<0.05$ was considered statistically significant.

\section{Results:-}

The age, weight, height, BMI, SBP, DBP, HR, and RR were comparable among the T2DM patients and controls. The FBS of the T2DM patients were significantly more $(p<0.05)$ than that of the controls $($ Table 1$)$. The T2DM patients had PPBS of $259.69 \pm 82.10 \mathrm{mg} / \mathrm{dl}$ and $\mathrm{HbA} 1 \mathrm{C}$ of $7.15 \pm 1.10 \%$.

Table 1:- Anthropometric, cardio-respiratory and FBS variables in T2DM and healthy controls.

\begin{tabular}{|c|c|c|c|}
\hline \multirow[t]{2}{*}{ Parameters } & \multicolumn{2}{|c|}{ Mean \pm SD } & \multirow[t]{2}{*}{ p value } \\
\hline & T2DM patients $(n=35)$ & Controls $(n=35)$ & \\
\hline Age (year) & $52.87 \pm 2.6$ & $53.43 \pm 2.75$ & 0.387 \\
\hline Weight (Kg) & $63.09 \pm 5.82$ & $64.17 \pm 6.88$ & 0.477 \\
\hline Height $(\mathrm{cm})$ & $163.69 \pm 8.32$ & $166.21 \pm 8.02$ & 0.200 \\
\hline $\mathrm{BMI}\left(\mathrm{Kg} / \mathrm{m}^{2}\right)$ & $23.54 \pm 1.92$ & $23.09 \pm 1.33$ & 0.250 \\
\hline SBP $(\mathrm{mm} \mathrm{Hg})$ & $120.05 \pm 5.38$ & $119.98 \pm 5.58$ & 0.957 \\
\hline $\mathrm{DBP}(\mathrm{mm} \mathrm{Hg})$ & $74.7 \pm 4.92$ & $73.95 \pm 4.77$ & 0.515 \\
\hline HR (beats per min) & $79.94 \pm 5.87$ & $79.91 \pm 6.64$ & 0.987 \\
\hline RR (per min) & $13.70 \pm 1.78$ & $13.86 \pm 1.79$ & 0.699 \\
\hline FBS & $167.21 \pm 54.17$ & $92.14 \pm 10.78$ & 0.001 \\
\hline
\end{tabular}

During hyperventilation, the T2DM patients had significantly more delta and beta activity $(\mathrm{p}<0.05)$ across all the scalp electrode sites (Table 2). During post-hyperventilation, the delta activity was significantly more $(\mathrm{p}<0.05)$ in the T2DM patients at midline frontal (Fz), right parietal (P4), right occipital (O2), left anterior frontal (Fp1), left temporal (T5), and left occipital (O1) regions (Table 3). The T2DM patients had significantly more beta activity (p < 0.05) at midline (Fz, Cz, Pz), bilateral frontal (Fp1, Fp2, F3, F4, F7, F8), left temporal (T3, T5), bilateral central $(\mathrm{C} 3, \mathrm{C} 4)$, and left parietal $(\mathrm{P} 3)$ regions (Table 3$)$. 
Table 2:- EEG power spectra in T2DM patients and healthy controls during hyperventilation.

\begin{tabular}{|c|c|c|c|c|c|c|c|c|c|c|c|}
\hline \multirow{2}{*}{$\begin{array}{l}\text { Electrode } \\
\text { Site }\end{array}$} & \multirow[b]{2}{*}{ Group } & \multicolumn{2}{|c|}{ Delta $\left(\mu V^{2}\right)$} & \multicolumn{2}{|c|}{ Theta $\left(\mu V^{2}\right)$} & \multicolumn{2}{|c|}{ Alpha1 $\left(\mu V^{2}\right)$} & \multicolumn{2}{|c|}{$\operatorname{Alpha2}\left(\mu V^{2}\right)$} & \multicolumn{2}{|c|}{ Beta $\left(\mu V^{2}\right)$} \\
\hline & & $\begin{array}{c}\text { Mean } \pm \\
\text { SD }\end{array}$ & $\begin{array}{c}\mathbf{p} \\
\text { value }\end{array}$ & $\begin{array}{c}\text { Mean } \pm \\
\text { SD }\end{array}$ & $\begin{array}{c}\mathbf{p} \\
\text { value }\end{array}$ & $\begin{array}{c}\text { Mean } \pm \\
\text { SD }\end{array}$ & $\begin{array}{c}\mathbf{p} \\
\text { value }\end{array}$ & $\begin{array}{c}\text { Mean } \pm \\
\text { SD }\end{array}$ & $\begin{array}{c}\mathbf{p} \\
\text { value }\end{array}$ & $\begin{array}{c}\text { Mean } \pm \\
\text { SD }\end{array}$ & $\begin{array}{c}\mathbf{p} \\
\text { value }\end{array}$ \\
\hline \multirow{2}{*}{ Fz } & T2DM & $1.87 \pm 0.23$ & \multirow{2}{*}{0.000} & $1.51 \pm 0.26$ & \multirow{2}{*}{0.893} & $1.50 \pm 0.48$ & \multirow{2}{*}{0.184} & $1.54 \pm 0.40$ & \multirow{2}{*}{0.088} & $1.50 \pm 0.22$ & \multirow{2}{*}{0.001} \\
\hline & Control & $1.64 \pm 0.28$ & & $1.50 \pm 0.36$ & & $1.65 \pm 0.44$ & & $1.34 \pm 0.57$ & & $0.96 \pm 0.62$ & \\
\hline \multirow{2}{*}{$\mathrm{Cz}$} & $\mathrm{T} 2 \mathrm{DM}$ & $1.91 \pm 0.21$ & \multirow{2}{*}{0.000} & $1.60 \pm 0.31$ & \multirow{2}{*}{0.822} & $1.50 \pm 0.44$ & \multirow{2}{*}{0.060} & $1.64 \pm 0.41$ & \multirow{2}{*}{0.114} & $1.53 \pm 0.17$ & \multirow{2}{*}{0.001} \\
\hline & $\mathrm{T} 2 \mathrm{DM}$ & $1.66 \pm 0.26$ & & $1.62 \pm 0.31$ & & $1.69 \pm 0.43$ & & $1.43 \pm 0.65$ & & $1.13 \pm 0.61$ & \\
\hline \multirow{2}{*}{$\mathbf{P z}$} & T2DM & $1.86 \pm 0.22$ & \multirow{2}{*}{0.000} & $1.44 \pm 0.22$ & \multirow{2}{*}{0.372} & $1.54 \pm 0.56$ & \multirow{2}{*}{0.098} & $1.53 \pm 0.50$ & 0253 & $1.53 \pm 0.20$ & 0.003 \\
\hline & Control & $1.58 \pm 0.28$ & & $1.50 \pm 0.32$ & & $1.74 \pm 0.45$ & & $1.69 \pm 0.66$ & 0.253 & $1.12 \pm 0.73$ & \\
\hline & T2DM & $1.90 \pm 0.31$ & Pen & $1.05 \pm 0.33$ & 7077 & $1.49 \pm 0.63$ & (1) & $1.21 \pm 0.32$ & & $1.31 \pm 0.17$ & 0004 \\
\hline Fp2 & Control & $1.67 \pm 0.29$ & 0.002 & $1.19 \pm 0.34$ & 0.071 & $1.48 \pm 0.49$ & 0.931 & $1.11 \pm 0.56$ & 0.373 & $0.95 \pm 0.70$ & 0.004 \\
\hline Fe & $\mathrm{T} 2 \mathrm{DM}$ & $1.48 \pm 0.33$ & 0001 & $0.84 \pm 0.46$ & 0115 & $1.65 \pm 1.93$ & 0,003 & $0.96 \pm 0.38$ & 0157 & $1.11 \pm 0.38$ & \\
\hline 58 & Control & $1.20 \pm 0.37$ & 0.001 & $0.68 \pm 0.36$ & 0.115 & $1.06 \pm 0.53$ & 0.093 & $0.80 \pm 0.57$ & $0.15 /$ & $0.72 \pm 0.79$ & 0.009 \\
\hline & $\mathrm{T} 2 \mathrm{DM}$ & $1.26 \pm 0.23$ & & $0.93 \pm 0.33$ & 0188 & $1.30 \pm 0.63$ & 0145 & $1.14 \pm 0.49$ & 0087 & $1.32 \pm 0.50$ & \\
\hline T4 & Control & $1.05 \pm 0.32$ & 0.002 & $0.84 \pm 0.20$ & 0.188 & $1.11 \pm 0.43$ & 0.143 & $0.93 \pm 0.51$ & 0.081 & $0.83 \pm 0.74$ & 0.002 \\
\hline & $\mathrm{T} 2 \mathrm{DM}$ & $1.39 \pm 0.29$ & & $0.87 \pm 0.41$ & & $1.47 \pm 0.63$ & & $1.30 \pm 0.45$ & & $1.31 \pm 0.37$ & \\
\hline T6 & Control & $1.07 \pm 0.48$ & 0.001 & $1.01 \pm 0.31$ & 0.124 & $1.52 \pm 0.65$ & 0.1713 & $1.13 \pm 0.63$ & 0.181 & $0.88 \pm 0.64$ & .001 \\
\hline F4 & $\mathrm{T} 2 \mathrm{DM}$ & $1.74 \pm 0.26$ & 0000 & $1.21 \pm 0.25$ & & $1.51 \pm 0.55$ & & $1.35 \pm 0.37$ & 0100 & $1.57 \pm 0.49$ & 0001 \\
\hline 14 & Control & $1.46 \pm 0.35$ & 0.000 & $1.16 \pm 0.19$ & & $1.49 \pm 0.50$ & $0.8 / 2$ & $1.14 \pm 0.65$ & 0.100 & $0.96 \pm 0.66$ & 0.001 \\
\hline C4 & $\mathrm{T} 2 \mathrm{DM}$ & $1.72 \pm 0.22$ & 0000 & $1.67 \pm 0.68$ & 0401 & $1.46 \pm 0.48$ & $0>60$ & $1.48 \pm 0.40$ & (104 & $1.39 \pm 0.40$ & \\
\hline $\mathrm{C} 4$ & Control & $1.45 \pm 0.30$ & 0.000 & $1.55 \pm 0.50$ & 0.401 & $1.59 \pm 0.46$ & 0.269 & $1.30 \pm 0.51$ & 0.104 & $0.95 \pm 0.53$ & 0.001 \\
\hline & $\mathrm{T} 2 \mathrm{DM}$ & $1.79 \pm 0.32$ & & $1.41 \pm 0.36$ & 0047 & $1.69 \pm 0.49$ & & $1.59 \pm 0.44$ & & $1.29 \pm 0.40$ & \\
\hline $\mathbf{P 4}$ & Control & $1.43 \pm 0.33$ & 0.000 & $1.41 \pm 0.41$ & 0.947 & $1.65 \pm 0.51$ & 0.738 & $1.41 \pm 0.43$ & $0.0 / 4$ & $0.96 \pm 0.51$ & 0.003 \\
\hline & T2DM & $1.78 \pm 0.26$ & & $1.05 \pm 0.44$ & & $1.71 \pm 0.52$ & & $1.49 \pm 0.44$ & & $1.60 \pm 0.51$ & \\
\hline & Control & $1.45 \pm 0.28$ & 0.000 & $1.21 \pm 0.26$ & 0.066 & $1.81 \pm 0.56$ & 0.420 & $1.55 \pm 0.74$ & 0.672 & $1.16 \pm 0.74$ & 0.005 \\
\hline Fn1 & T2DM & $1.91 \pm 0.30$ & 0004 & $1.42 \pm 0.35$ & 0143 & $1.30 \pm 0.54$ & 0134 & $1.20 \pm 0.31$ & 0587 & $1.50 \pm 0.40$ & 0003 \\
\hline Fp1 & Control & $1.71 \pm 0.27$ & 0.004 & $1.31 \pm 0.29$ & 0.143 & $1.47 \pm 0.43$ & 0.134 & $1.28 \pm 0.72$ & 0.581 & $1.07 \pm 0.70$ & 0.003 \\
\hline F7 & $\mathrm{T} 2 \mathrm{DM}$ & $1.45 \pm 0.28$ & 0001 & $0.80 \pm 0.27$ & 0072 & $1.07 \pm 0.49$ & 0497 & $0.93 \pm 0.36$ & 0794 & $1.33 \pm 1.20$ & 000 \\
\hline F7 & Control & $1.21 \pm 0.30$ & 0.001 & $0.71 \pm 0.16$ & 0.072 & $1.00 \pm 0.41$ & 0.497 & $0.89 \pm 0.74$ & 0.794 & $0.65 \pm 0.59$ & 0.004 \\
\hline$T 2$ & $\mathrm{~T} 2 \mathrm{DM}$ & $1.23 \pm 0.24$ & 016 & $0.82 \pm 0.42$ & 0064 & $1.03 \pm 0.42$ & 0005 & $1.18 \pm 0.48$ & 0325 & $1.38 \pm 0.69$ & \\
\hline 13 & Control & $1.07 \pm 0.30$ & 0.016 & $1.02 \pm 0.43$ & 0.061 & $1.03 \pm 0.37$ & 0.995 & $1.03 \pm 0.79$ & 0.335 & $0.81 \pm 0.64$ & 0.001 \\
\hline & $\mathrm{T} 2 \mathrm{DM}$ & $1.39 \pm 0.25$ & & $1.06 \pm 0.42$ & & $1.54 \pm 0.75$ & 0460 & $1.31 \pm 0.44$ & & $1.23 \pm 0.61$ & \\
\hline 15 & Control & $1.07 \pm 0.46$ & 0.001 & $1.13 \pm 0.48$ & $0.50 /$ & $1.42 \pm 0.65$ & 0.469 & $1.41 \pm 0.83$ & 0.528 & $0.78 \pm 0.58$ & 0.002 \\
\hline F3 & $\mathrm{T} 2 \mathrm{DM}$ & $1.72 \pm 0.25$ & 0.001 & $1.26 \pm 0.24$ & 0.952 & $1.46 \pm 0.82$ & 0759 & $1.33 \pm 0.36$ & 0412 & $1.51 \pm 0.52$ & 0.002 \\
\hline & Control & $1.50 \pm 0.28$ & & $1.26 \pm 0.24$ & & $1.41 \pm 0.43$ & & $1.48 \pm 1.05$ & & $0.98 \pm 0.81$ & \\
\hline $\mathrm{Cl}_{3}$ & T2DM & $1.67 \pm 0.21$ & 0000 & $1.26 \pm 0.29$ & 0260 & $1.49 \pm 0.49$ & 0650 & $1.31 \pm 0.71$ & 0524 & $1.61 \pm 0.61$ & 0005 \\
\hline $\mathrm{C3}$ & Control & $1.43 \pm 0.30$ & 0.000 & $1.34 \pm 0.33$ & 0.269 & $1.54 \pm 0.49$ & 0.650 & $1.43 \pm 0.91$ & 0.524 & $1.08 \pm 0.89$ & 0.000 \\
\hline $\mathbf{D 3}$ & T2DM & $1.67 \pm 0.19$ & & $1.24 \pm 0.35$ & & $1.49 \pm 0.44$ & & $1.37 \pm 0.74$ & & $1.45 \pm 0.50$ & \\
\hline P3 & Control & $1.39 \pm 0.41$ & 0.001 & $1.32 \pm 0.36$ & 0.381 & $1.63 \pm 0.56$ & & $1.64 \pm 1.00$ & 0.20 & $0.99 \pm 0.60$ & 0.001 \\
\hline & T2DM & $1.74 \pm 0.29$ & & $1.18 \pm 0.29$ & & $1.51 \pm 0.50$ & & $1.36 \pm 0.86$ & & $1.32 \pm 0.26$ & \\
\hline & Control & $1.41 \pm 0.26$ & 0.000 & $1.29 \pm 0.42$ & 195 & $1.70 \pm 0.57$ & & $1.43 \pm 0.65$ & & $1.13 \pm 0.61$ & \\
\hline
\end{tabular}

$\mu \mathrm{V}^{2}$, square microvolt; SD, Standard Deviation; $\mathrm{p}<0.05$ considered statistically significant 
Table 3:- EEG power spectra in T2DM patients and healthy controls during post-hyperventilation.

\begin{tabular}{|c|c|c|c|c|c|c|c|c|c|c|c|}
\hline \multirow{2}{*}{$\begin{array}{l}\text { Electrode } \\
\text { Site }\end{array}$} & \multirow[b]{2}{*}{ Group } & \multicolumn{2}{|c|}{ Delta $\left(\mu V^{2}\right)$} & \multicolumn{2}{|c|}{ Theta $\left(\mu \mathbf{V}^{2}\right)$} & \multicolumn{2}{|c|}{ Alpha1 $\left(\mu V^{2}\right)$} & \multicolumn{2}{|c|}{ Alpha2 $\left(\mu \mathbf{V}^{2}\right)$} & \multicolumn{2}{|c|}{ Beta $\left(\mu V^{2}\right)$} \\
\hline & & $\begin{array}{c}\text { Mean } \pm \\
\text { SD }\end{array}$ & $\begin{array}{c}\mathbf{p} \\
\text { value }\end{array}$ & $\begin{array}{c}\text { Mean } \pm \\
\text { SD }\end{array}$ & $\begin{array}{c}\mathbf{p} \\
\text { value }\end{array}$ & $\begin{array}{c}\text { Mean } \pm \\
\text { SD }\end{array}$ & $\begin{array}{c}\mathbf{p} \\
\text { value }\end{array}$ & $\begin{array}{c}\text { Mean } \pm \\
\text { SD }\end{array}$ & $\begin{array}{c}\mathbf{p} \\
\text { value }\end{array}$ & $\begin{array}{c}\text { Mean } \pm \\
\text { SD }\end{array}$ & $\begin{array}{c}\mathbf{p} \\
\text { value }\end{array}$ \\
\hline \multirow{2}{*}{$\mathbf{F z}$} & T2DM & $1.79 \pm 0.23$ & \multirow{2}{*}{0.041} & $1.39 \pm 0.29$ & \multirow{2}{*}{0.683} & $1.50 \pm 0.49$ & \multirow{2}{*}{0.617} & $1.26 \pm 0.38$ & \multirow{2}{*}{0.096} & $1.54 \pm 0.45$ & \multirow{2}{*}{0.000} \\
\hline & Control & $1.64 \pm 0.34$ & & $1.36 \pm 0.28$ & & $1.56 \pm 0.50$ & & $1.10 \pm 0.41$ & & $0.95 \pm 0.46$ & \\
\hline \multirow{2}{*}{$\mathrm{Cz}$} & T2DM & $1.80 \pm 0.34$ & \multirow{2}{*}{0.163} & $1.43 \pm 0.32$ & \multirow{2}{*}{0.893} & $1.62 \pm 0.45$ & \multirow{2}{*}{0.527} & $1.36 \pm 0.41$ & \multirow{2}{*}{0.508} & $1.64 \pm 0.43$ & \multirow{2}{*}{0.000} \\
\hline & Control & $1.68 \pm 0.37$ & & $1.44 \pm 0.28$ & & $1.69 \pm 0.49$ & & $1.30 \pm 0.39$ & & $1.09 \pm 0.50$ & \\
\hline \multirow[b]{2}{*}{$\mathbf{P z}$} & T2DM & $1.79 \pm 0.30$ & \multirow{2}{*}{0.092} & $1.29 \pm 0.40$ & \multirow{2}{*}{0.602} & $1.76 \pm 0.52$ & \multirow{2}{*}{0.339} & $1.50 \pm 0.48$ & 0,100 & $1.51 \pm 0.24$ & 002 \\
\hline & Control & $1.66 \pm 0.33$ & & $1.33 \pm 0.25$ & & $1.64 \pm 0.56$ & & $1.43 \pm 0.44$ & 0.489 & $1.17 \pm 0.60$ & 0.003 \\
\hline & $\mathrm{T} 2 \mathrm{DM}$ & $1.78 \pm 0.26$ & 0074 & $1.02 \pm 0.45$ & & $1.30 \pm 0.48$ & & $1.06 \pm 0.37$ & & $1.31 \pm 0.27$ & \\
\hline F 2 & Control & $1.64 \pm 0.38$ & 0.014 & $1.15 \pm 0.24$ & 0.120 & $1.39 \pm 0.55$ & $0.4 \% 0$ & $0.92 \pm 0.39$ & 0.110 & $0.83 \pm 0.41$ & 0.000 \\
\hline Fo & $\mathrm{T} 2 \mathrm{DM}$ & $1.31 \pm 0.43$ & 0067 & $0.42 \pm 0.63$ & 005 & $0.82 \pm 0.56$ & $0<0$ & $0.63 \pm 0.37$ & 0110 & $0.91 \pm 0.27$ & 000 \\
\hline & Control & $1.31 \pm 0.59$ & 0.907 & $0.68 \pm 0.29$ & 0.004 & $0.87 \pm 0.57$ & 0.090 & $0.49 \pm 0.37$ & 0.105 & $0.51 \pm 0.51$ & 0.000 \\
\hline & $\mathrm{T} 2 \mathrm{DM}$ & $1.27 \pm 0.24$ & 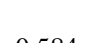 & $0.61 \pm 0.43$ & & $0.89 \pm 0.51$ & & $0.81 \pm 0.39$ & & $0.94 \pm 0.27$ & \\
\hline & Control & $1.22 \pm 0.44$ & & $0.76 \pm 0.29$ & 0.070 & $0.98 \pm 0.55$ & & $0.74 \pm 0.37$ & & $0.79 \pm 0.53$ & \\
\hline & T2DM & $1.34 \pm 0.22$ & & $0.88 \pm 0.33$ & & $1.16 \pm 0.63$ & & $1.03 \pm 0.48$ & & $1.07 \pm 0.23$ & \\
\hline 10 & Control & $1.19 \pm 0.52$ & 0.135 & $0.87 \pm 0.35$ & 0.881 & $1.36 \pm 0.71$ & 0.221 & $0.97 \pm 0.46$ & 0.614 & $0.87 \pm 0.66$ & 0.101 \\
\hline & $\mathrm{T} 2 \mathrm{DM}$ & $1.64 \pm 0.24$ & & $1.12 \pm 0.33$ & & $1.33 \pm 0.50$ & & $1.09 \pm 0.37$ & & $1.49 \pm 0.59$ & \\
\hline 14 & Control & $1.58 \pm 0.45$ & 0.474 & $1.13 \pm 0.28$ & 0.834 & $1.33 \pm 0.55$ & 0.909 & $0.93 \pm 0.38$ & 0.085 & $0.82 \pm 0.45$ & 0.000 \\
\hline 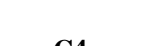 & $\mathrm{T} 2 \mathrm{DM}$ & $1.62 \pm 0.34$ & 074 & $1.17 \pm 0.37$ & ( & $1.47 \pm 0.46$ & 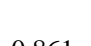 & $1.28 \pm 0.43$ & & $1.51 \pm 0.41$ & \\
\hline 4 & Control & $1.60 \pm 0.36$ & & $1.24 \pm 0.28$ & (.JJ2 & $1.49 \pm 0.53$ & & $1.20 \pm 0.38$ & & $0.98 \pm 0.44$ & 0.000 \\
\hline & T2DM & $1.91 \pm 0.49$ & & $1.19 \pm 0.27$ & & $1.58 \pm 0.48$ & & $1.39 \pm 0.48$ & & $1.39 \pm 0.24$ & \\
\hline 15 & Control & $1.54 \pm 0.44$ & 0.001 & $1.19 \pm 0.28$ & 0.991 & $1.48 \pm 0.62$ & 0.440 & $1.30 \pm 0.42$ & 0.509 & $1.20 \pm 0.74$ & 0.109 \\
\hline & T2DM & $1.66 \pm 0.24$ & & $1.25 \pm 0.29$ & & $1.48 \pm 0.50$ & & $1.41 \pm 0.50$ & & $1.23 \pm 0.35$ & \\
\hline U & Control & $1.50 \pm 0.38$ & 0.050 & $1.20 \pm 0.30$ & 0.420 & $1.67 \pm 0.61$ & 0.150 & $1.35 \pm 0.50$ & $0.5 / 2$ & $1.21 \pm 0.81$ & $0.86 /$ \\
\hline & $\mathrm{T} 2 \mathrm{DM}$ & $1.92 \pm 0.23$ & & $1.11 \pm 0.37$ & & $1.35 \pm 0.48$ & & $1.09 \pm 0.37$ & & $1.40 \pm 0.39$ & \\
\hline Fр1 & Control & $1.68 \pm 0.37$ & 0.002 & $1.18 \pm 0.23$ & 0.508 & $1.43 \pm 0.60$ & 0.508 & $0.91 \pm 0.39$ & 0.039 & $0.73 \pm 0.47$ & 0.000 \\
\hline & T2DM & $1.35 \pm 0.28$ & & $0.76 \pm 0.38$ & & $0.85 \pm 0.53$ & & $0.60 \pm 0.37$ & & $1.13 \pm 0.77$ & \\
\hline 57 & Control & $1.22 \pm 0.35$ & 0.005 & $0.70 \pm 0.26$ & 0.400 & $0.90 \pm 0.54$ & 0.070 & $0.47 \pm 0.34$ & 0.120 & $0.44 \pm 0.59$ & 0.000 \\
\hline & T2DM & $1.17 \pm 0.35$ & & $0.83 \pm 0.41$ & 1 & $0.98 \pm 0.43$ & & $0.80 \pm 0.42$ & & $1.13 \pm 0.36$ & \\
\hline & Control & $1.15 \pm 0.34$ & 0.190 & $0.71 \pm 0.29$ & & $0.93 \pm 0.56$ & & $0.74 \pm 0.38$ & & $0.57 \pm 0.52$ & 0.000 \\
\hline & T2DM & $1.29 \pm 0.19$ & & $0.98 \pm 0.65$ & & $1.22 \pm 0.56$ & & $1.00 \pm 0.48$ & & $1.14 \pm 0.31$ & \\
\hline 15 & Control & $1.15 \pm 0.30$ & 0.019 & $0.80 \pm 0.35$ & 0.145 & $1.33 \pm 0.76$ & 0.501 & $0.96 \pm 0.54$ & 0.124 & $0.83 \pm 0.45$ & 0.001 \\
\hline & T2DM & $1.62 \pm 0.28$ & 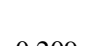 & $1.13 \pm 0.29$ & & $1.31 \pm 0.46$ & & $1.06 \pm 0.39$ & & $1.74 \pm 1.30$ & \\
\hline 53 & Control & $1.53 \pm 0.34$ & 0.209 & $1.09 \pm 0.26$ & 0.536 & $1.26 \pm 0.57$ & 0.637 & $0.87 \pm 0.42$ & 0.053 & $0.72 \pm 0.51$ & 0.000 \\
\hline & T2DM & $1.62 \pm 0.24$ & & $1.21 \pm 0.33$ & & $1.56 \pm 0.52$ & & $1.24 \pm 0.45$ & & $1.68 \pm 0.91$ & \\
\hline 4 & Control & $1.53 \pm 0.34$ & 0.171 & $1.15 \pm 0.27$ & $0.4+2$ & $1.38 \pm 0.59$ & 0.175 & $1.18 \pm 0.47$ & (.50J & $0.85 \pm 0.49$ & 0.000 \\
\hline & T2DM & $1.58 \pm 0.24$ & & $1.22 \pm 0.34$ & & $1.73 \pm 0.73$ & & $1.39 \pm 0.48$ & & $1.46 \pm 0.44$ & \\
\hline 15 & Control & $1.48 \pm 0.31$ & & $1.12 \pm 0.27$ & & $1.46 \pm 0.66$ & 0.104 & $1.28 \pm 0.51$ & 0.3 & $0.96 \pm 0.48$ & 0.000 \\
\hline 01 & T2DM & $1.63 \pm 0.20$ & & $1.23 \pm 0.40$ & & $1.58 \pm 0.49$ & & $1.39 \pm 0.47$ & & $1.27 \pm 0.20$ & \\
\hline & Control & $1.68 \pm 0.37$ & & $1.44 \pm 0.28$ & 0.035 & $1.69 \pm 0.49$ & 0.950 & $1.30 \pm 0.39$ & 0.031 & $1.09 \pm 0.50$ & 0.191 \\
\hline
\end{tabular}

$\mu \mathrm{V}^{2}$, square microvolt; SD, Standard Deviation; $\mathrm{p}<0.05$ considered statistically significant 


\section{Discussion:-}

Our study revealed a diffuse increase in low-frequency slow waves and high-frequency fast waves in EEG in T2DM patients on provoking their brain activity by voluntary HV. In addition, there was prolonged existence of lowfrequency slow waves at few brain regions and high-frequency fast waves in some brain regions in the EEG after cessation of HV during post-HV.

The studies have shown that the EEG power spectra of delta and beta activity are more in T2DM patients at most of the brain regions $(8,9)$ and hyperventilation helps to increase the yield of EEG. Diffuse EEG changes characteristically occur in metabolic encephalopathies unless there is a pre-existing or concomitant structural lesion. Diffuse slowing of the EEG has been described in hyperglycemia or hypoglycemia, Addison's disease, hypopituitarism, pulmonary failure, and hyperparathyroidism. These slow waves initially may be episodic or paroxysmal and are enhanced by hyperventilation (10).

The uncontrolled diabetes mellitus can facilitate the occurrence of watershed ischemia and the EEG is slow and severely disorganized with diffusely disordered background activity (11). A meta-analysis of 24 studies showed an association of depression with hyperglycemia in T2DM (12). The elevated depressive symptoms have been associated with a higher risk of development of T2DM (13). Diabetes may place patients at risk for depression through a biological mechanism linking the metabolic changes of DM to changes in the CNS (14). There exists a common point of pathophysiology between MDD and DM (15). The prevalence of anxiety and depression symptoms in patients with diabetes was more than double the general population estimates (16). The elevated slowwave activity as those observed in DM has been demonstrated in MDD patients (9). Kwon et al (17) found increased low-frequency EEG activity, including theta and delta power over the right hemisphere in depressed patients compared with age-matched healthy controls.

Hyperglycemia is associated with various neurological manifestations, including encephalopathy (18). The features of encephalopathy in hyperglycemia vary depending on the degrees of hyperglycemia and hyperosmolarity. The hyperglycemic encephalopathy has variable EEG findings that range from normal, at serum glucose $<400 \mathrm{mg} / \mathrm{dl}$, to the initial appearance of mixed slow and fast activity with some intermingled spiking. However, diffuse and oftencontinuous medium to high voltage theta-delta activity predominates with increasing serum glucose concentrations (20).

In the study done by Khan et al. (21) in 889 DM patients, they identified $57.9 \%$ and $43.5 \%$ positive for anxiety and depression respectively. Anxiety disorders are also highly prevalent in DM patients (22). The rate of clinical anxiety with co-morbid affective disorder is higher than expected in DM patients, although the reason is not clear (23). Lustman et al. (24) had also observed high rates of generalized anxiety disorders in DM patients. Beta waves (faster than 13/sec) are usually seen, especially on the frontal and central areas, in tense and anxious patients (25). Cohn (26) noted frontal beta activity (18-22/sec) in patients suffering from anxiety states.

Several activation methods, including hyperventilation, have been used in clinical EEG recording to enhance preexisting abnormalities and/or induce abnormal findings in otherwise normal EEGs (27). In our study, the hyperventilation might have enhanced the EEG manifestations of preexisting CNS abnormalities in T2DM patients. Apart from hyperglycemia, our results might have been affected by contemporary depression and anxiety or hyperglycemic encephalopathy in our diabetic patients. However, we did not assess the depression and anxiety or hepatic encephalopathy by using any other tools or neuroimaging techniques in our subjects.

\section{Conclusion:-}

The T2DM patients revealed a diffuse increase in low-frequency slow waves and high-frequency fast waves at delta and beta ranges respectively on EEG recording during hyperventilation, a classic activation method. In addition, there was prolonged existence of the responses in EEG at various sites after cessation of HV. Our findings support the belief that T2DM patients manifest CNS abnormalities as evidenced by the increased EEG activity in lowfrequency slow waves and high-frequency fast waves. However, further collaborative studies including neurophysiological tests, Folstein (Mini-Mental State Examination) test, and neuroimaging techniques are needed to evident a link of DM with neuropsychiatric/cerebral disorders.

\section{Acknowledgement:-}

All the patients, their relatives, and healthy individuals are thankfully acknowledged for their active participation in the study. 


\section{References:-}

1. International Diabetes Federation: IDF Diabetes Atlas, $7^{\text {th }}$ ed. Brussels, Belgium. www.diabetesatlas.org (updated 2015).

2. Powers AC. Harrison's principles of internal medicine. $19^{\text {th }}$ ed. New York: The McGraw-Hill Companies, Inc; 2015. Chapter 419, Diabetes Mellitus: Complications; p2422-30.

3. Biessels GJ, Kappelle AC, Bravenboer B, Erkelens DW, Gispen WH. Cerebral function in diabetes mellitus. Diabetologia 1994;37:643-50.

4. Pan A, Lucas M, Sun Q, van Dam RM, Franco OH, Manson JE, Willet WC, Ascherio A, Hu FB. Bidirectional association between depression and type 2 diabetes mellitus in women. Arch Intern Med 2010;170(21):1884-91.

5. Balhara YPS. Diabetes and psychiatric disorders. Indian J Endocrinol Metab 2011;15(4):274-83.

6. Paudel BH, Limbu N, Panta R, Ghimire N, Shrestha B, Deo S. Neurophysiology Application Notes. Dharan: Physiological Society of Nepal; 2012. Chapter 1, Electroencephalography; p7-18.

7. Duffy FH, Iyer VG, Surwillo WW. Clinical electroencephalography and topographic brain mapping. New York: Springer-Verlag Inc; 1989. Chapter 1, Brain Electrical Activity: An Introduction to EEG Recording; p110.

8. Panta R, Khadka R, Thakur D, Limbu N, Paudel BH. Which occurs first in patients with type 2 diabetes mellitus? Central or peripheral neuropathy. Int Arch BioMed Clin Res 2016;2(2):73-8.

9. Baskaran A, Milev R, McIntyre RS. A review of electroencephalographic changes in diabetes mellitus in relation to major depressive disorder. Neuropsychiatr Dis 2013;9:143-50.

10. Aminoff MJ. Electrodiagnosis in clinical neurology. Philadelphia: Elsevier Inc; 2005. Chapter 3, Electroencephalography: General Principles and Clinical Applications; p37-84.

11. Naquet R, Vigouroux RA. EEG Handbook of electroencephalography and clinical neurophysiology. Vol 14(A). Amsterdam: Elsevier Inc; 1972. p38-84.

12. Lustman P, Anderson RJ, Freedland KE, de Groot M, Carney RM, Clouse RE. Depression and poor glycemic control: a meta-analytic review of the literature. Diabetes Care 2000;23:934-42.

13. Demakakos P, Pierce MB, Hardy R. Depressive symptoms and risk of type 2 diabetes in a national sample of middle-aged and older adults: the English longitudinal study of aging. Diabetes Care 2010;33(4):792-97.

14. McIntyre RS, Soczynska JK, Konarski JZ, Woldeyohannes HO, Law CW, Miranda A, Fulgosi D, Kennedy SH. Should depressive syndromes be reclassified as "metabolic syndrome type II"? Ann Clin Psychiatry 2007;19(4):257-64.

15. McIntyre RS, Kenna HA, Nguyen HT, Law CW, Sultan F, Woldeyohannes HO, Adams AK, ChengJS, Lourenco M, Kennedy SH, Rasgon NL. Brain volume abnormalities and neurocognitive deficits in diabetes mellitus: points of pathophysiological commonality with mood disorders? Adv Ther 2010;27(2):63-80.

16. Collins MM, Corcoran P, and Perry IJ. Anxiety and depression symptoms in patients with diabetes. Diabet Med 2009;26:153-61.

17. Kwon JS, Youn T, Jung HY. Right hemisphere abnormalities in major depression: quantitative electroencephalographic findings before and after treatment. J Affect Disord 1996;40(3):169-73.

18. Maccario M, Messis CP, Vastola EF. Focal seizures as a manifestation of hyperglycemia without ketoacidosis. A report of seven cases with review of the literature. Neurology 1965;15:195-206.

19. Gibbs FA, Williams D, Gibbs EL. Modification of the cortical frequency spectrum by changes in $\mathrm{CO}_{2}$, blood sugar, and $\mathrm{O}_{2}$. J Neurophysiol 1940;3:49-58.

20. Maccario M. Neurological dysfunction associated with nonketotic hyperglycemia. Arch Neurol 1968;19:52534.

21. Khan KA, Lalani S, Dhanani R, Iqbal SA, Rafique G, White F. Anxiety and depression among outpatients with type 2 diabetes: A multi-centre study of prevalence and associated factors. Diabetol Metab Syndr 2010;2:72

22. Grigsby AB, Anderson RJ, Freedland KF, Couse FE, Lustman PJ. Prevalence of anxiety in adults with diabetes: a systematic review. J Psychosom Res 2002;53(6):1053-60.

23. Maier W, Falkai P. The epidemiology of comorbidity between depression, anxiety disorders, and somatic diseases. Int Clin Psychopharmacol 1999;14(Suppl 2):S1-6.

24. Lustman PJ, Griffith LS, Clouse RE, Cryer PE. Psychiatric illness in diabetes mellitus. Relationship to symptoms and glucose control. J Nerv Ment Dis 1986;174(12):736-42.

25. John R. Hughes. EEG in clinical practice. $2^{\text {nd }}$ ed. Boston: Butterworth-Heinemann; 1994; Chapter 2, Names of rhythms or patterns; p15-18.

26. Cohn R. The influence of emotions on the human electroencephalogram. J Nerv Ment Dis 1946;104:351-57.

27. Takahashi T. Electroencephalography: basic principles, clinical applications, and related field. $5^{\text {th }}$ ed. Philadelphia: Lippincott Williams \& Wilkins; 2005: Chapter 14, Activation methods; p241-62. 